\title{
Ein neuer Fonds gegen Armut und soziale Ausgrenzung
}

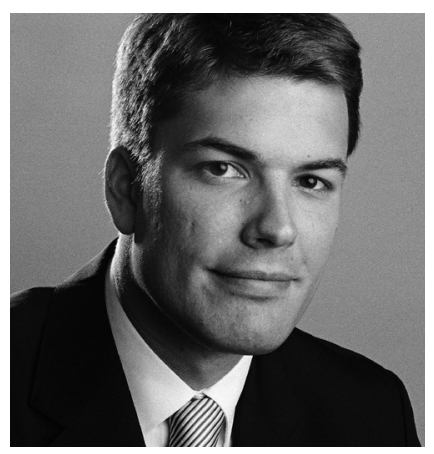

VON HENNING BRAEM Henning Braem ist als EU-Referent für die Bank für Sozialwirtschaft AG am Standort Brüssel tätig. Der Europa-Service der Bank für Sozialwirtschaft verfolgt die aktuellen europäischen Entwicklungen in der Sozial- und Gesundheitswirtschaft und bietet das EUFachinformationssystem (EUFIS) an. Dieses stellt seinen Nutzern ein umfassendes Informationsangebot zu den Themenfeldern Soziales und Beschäftigung, Gesundheit, Bildung und Jugend sowie zu EU-Förderprogrammen zur Verfügung www.eufis.eu

\author{
Die Europäische Kommission hat einen \\ Verordnungsvorschlag vorgelegt zu einem neuen \\ Hilfsfonds für die am stärksten von Armut betroffenen \\ Personen. Mit dem Geld sollen zwischen 2014 und 2020 \\ entsprechende Programme in den Mitgliedstaaten \\ finanziert werden. Für die Durchführung der Projekte \\ gebraucht werden Partnerorganisationen.
}

Der Verordnungsvorschlag der Europäischen Kommission für einen Europäischen Hilfsfonds für die am stärksten von Armut betroffenen Personen soll mitgliedstaatliche Programme finanzieren, die Nahrungsmittel, Kleidung und andere Güter des täglichen Bedarfs an obdachlose Menschen und in materieller Armut lebende Kinder verteilen sowie die soziale Wiedereingliederung der am stärksten unter Armut leidenden Menschen fördern.

Hintergrund des Vorschlags ist zum einen das Auslaufen des aktuellen EUNahrungsmittelhilfeprogramms Ende dieses Jahres. Dieses 1987 eingerichtete Hilfsprogramm diente ursprünglich dazu, die landwirtschaftliche Überproduktion sinnvoll zu verwenden und wurde so eine wichtige Quelle für Wohltätigkeits- und zivilgesellschaftliche Organisationen, um von Armut betroffene Menschen unterstützen zu können. Als Folge zahlreicher Reformen der gemeinsamen Agrarpolitik und dem erwarteten Abbau der Interventionsbestände fällt die Grundlage des Nahrungsmittelhilfeprogramms weg, so dass es Ende 2013 eingestellt wird.

Zum anderen hat die andauernde Wirtschaftskrise schwerwiegende soziale Auswirkungen. So waren im Jahr 2010 mit 116 Millionen Menschen fast ein Viertel der Europäer armutsgefährdet oder von sozialer Ausgrenzung bedroht. Das waren rund zwei Millionen mehr als im Vorjahr und die vorliegen- den Zahlen für 2011 bestätigen diesen Trend. Darunter leiden 40 Millionen Menschen unter gravierender materieller Armut (Deprivation). Von Obdachlosigkeit als besonders gravierende Form materieller Armut waren Schätzungen zufolge 2009/2010 in Europa 4,1 Millionen Menschen betroffen. In jüngster Zeit hat die Obdachlosigkeit auch aufgrund der sozialen Auswirkungen der wirtschaftlichen und sozialen Krise sowie steigender Arbeitslosigkeit zugenommen, dabei sind zunehmend Familien mit Kindern, junge Menschen und Menschen mit Migrationshintergrund betroffen. Zudem sind EU-weit 25,4 Millionen Kinder armutsgefährdet oder von sozialer Ausgrenzung bedroht.

Gleichzeitig haben sich durch die Wirtschaftskrise in vielen Mitgliedstaaten aufgrund finanzieller Zwänge die Möglichkeiten reduziert, diese Menschen adäquat zu unterstützen.

Angesichts dieser Entwicklung und im Hinblick auf das EU-2020-Armutsziel - Senkung der Anzahl der in Armut lebenden oder armutsgefährdeten Personen um mindestens 20 Millionen - soll der Europäische Hilfsfonds dem übergeordneten Ziel dienen, durch die wirksame Bekämpfung der Armut den sozialen Zusammenhalt in der Europäischen Union zu stärken.

Der neue Fonds soll das Nahrungsmittelhilfeprogramm ersetzen, aber auch darüber hinausgehen. So definiert der Verordnungsvorschlag den Fonds als 
umfassendes Instrument, das materielle Hilfe in Form von Nahrungsmitteln, Waren und sonstigen Gütern für $\mathrm{Ob}$ dachlose und für von materieller Armut betroffene Kinder fördert und mit flankierenden Maßnahmen kombiniert wird, die auf die soziale Wiedereingliederung der am stärksten von Armut betroffenen Personen abzielen.

Der Fonds soll demnach mitgliedstaatliche Programme finanzieren, durch die neben der Gewährleistung materieller Unterstützung mit dem Ziel des sozialen Zusammenhalts auch grundlegende Maßnahmen für die soziale Integration der Betroffenen durchgeführt werden. Die Mittel für solche flankierenden Maßnahmen können ebenfalls aus dem Fonds bereitgestellt werden.

Im Rahmen des vorgeschlagenen Fonds sollen die Mitgliedstaaten Unterstützung für operationelle Programme im Zeitraum 2014 bis 2020 beantragen und mit Hilfe von Partnerorganisationen die Mittel verteilen bzw. die begleitenden Integrationsmaßnahmen durchführen.

\section{Wie die Sozialwirtschaft eingebunden werden soll}

Als Partnerorganisationen benennt der Verordnungsvorschlag solche Organisationen, die Nahrungsmittel oder sonstige Güter direkt oder indirekt an die am stärksten von Armut betroffenen Personen abgeben. Damit der Fonds tatsächlich zu einer nachhaltigen Reduktion der Armut und zur Verbesserung des sozialen Zusammenhalts beiträgt, sollen die Partnerorganisationen, die die Nahrungsmittel oder sonstigen Güter direkt abgeben, selbst Aktivitäten durchführen, die die Bereitstellung materieller Unterstützung ergänzen und auf die soziale Integration der am stärksten von Armut betroffenen Personen abzielen.

Die mitgliedstaatlichen Behörden können die Fondsmittel verwenden, um entweder selbst Nahrungsmittel oder sonstige Güter einzukaufen und den Partnerorganisationen zur Verfügung zu stellen oder um die Partnerorganisationen mit den dafür notwendigen Mitteln auszustatten. Der Vorschlag sieht auch die Möglichkeit vor, Nahrungsmittel aus Interventionsbeständen zu verwenden. Auch wenn eine Partnerorganisation die Nahrungsmittel oder sonstige Güter kauft, kann sie diese entweder selbst verteilen oder die Verteilung anderen Partnerorganisationen übertragen.

Nach dem Vorschlag haben die mitgliedstaatlichen Behörden beträchtliche Flexibilität, um ihre Unterstützung so zu planen und zu organisieren, dass sie mit ihren nationalen Programmen in Einklang steht. Detaillierte Vergabekriterien für die Unterstützung sollen die Mitgliedstaaten oder auch die Partnerorganisationen ausarbeiten, da sie die Unterstützung am besten gezielt auf lokale Bedürfnisse abstimmen können.

Für den Fonds ist ein Budget von 2,5 Milliarden Euro aus der Mittelausstattung für die Strukturfonds für den Zeitraum 2014 bis 2020 vorgesehen. Die Kofinanzierung der mitgliedstaatlichen Programme durch den Fonds soll sich auf bis zu 85 Prozent belaufen, die verbleibende Finanzierungslücke ist durch die Mitgliedstaaten zu schließen. Auf Initiative oder im Auftrag der Europäischen Kommission durchgeführte Maßnahmen für technische Unterstützung können jedoch zu 100 Prozent durch den Fonds finanziert werden.

Als förderfähige Maßnahmen nennt der Vorschlag vor allem den Kauf von Nahrungsmitteln oder grundlegenden Konsumgütern für den persönlichen Gebrauch von obdachlosen Personen oder von Kindern. Darüber hinaus können die Partnerorganisationen Pauschalen in Höhe von jeweils fünf Prozent des für den Kauf dieser Güter anfallenden Betrages für die Kosten für Verwaltung, Transport- und Lagerkosten sowie für die Aktivitäten zur Förderung der sozialen Inklusion geltend machen. Durch dieses Pauschalierungssystem soll der Verwaltungsaufwand minimal gehalten und eine effektive Nutzung des Fonds ermöglicht werden.

\section{Wie der Hilfsfonds arbeiten soll}

Bei der Durchführung sollen die Mitgliedstaaten im Sinne eines möglichst geringen Verwaltungsaufwands die für den Europäischen Sozialfonds eingerichteten Strukturen und Behörden nutzen.

Die Bestimmungen für Planung, Monitoring, Evaluierung sowie Information und Kommunikation sind jedoch einfacher gefasst und damit den Zielen und Zielgruppen des Fonds angepasst. So sollen die Mitgliedstaaten unter anderem Informationen zu den aus dem Fonds geförderten Maßnahmen bereit- stellen und eine mindestens jährlich aktualisierte Liste der aus dem Fonds unterstützten Vorhaben über eine Website zugänglich machen. Zu Evaluierungszwecken sind die Mitgliedstaaten beispielsweise verpflichtet, der Kommission jährliche Durchführungsberichte zum operationellen Programm für das vorausgegangene Geschäftsjahr vorzulegen.

Der Verordnungsvorschlag wurde an das Europäische Parlament und den Rat weitergeleitet. Die Abstimmung im Plenum des Europäischen Parlaments ist für Juli 2013 vorgesehen.

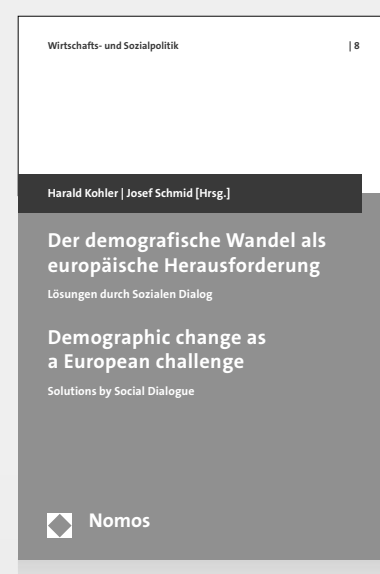

\section{Der demografische \\ Wandel als europäische Herausforderung. Demographic change as a European challenge}

Lösungen durch Sozialen Dialog. Solutions by Social Dialogue

Herausgegeben von Harald Kohler und Josef Schmid 2012, 232 S., brosch., 22,-€ ISBN 978-3-8329-6578-5

(Wirtschafts- und Sozialpolitik, Bd. 8)

www.nomos-shop.de/13584

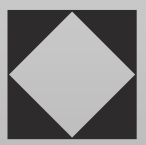

Nomos 